\title{
PREVALENCIA DE SOBREPESO Y OBESIDAD EN ADOLESCENTES DE UN DISTRITO URBANO DE LIMA, PERÚ 2012
}

\author{
Gaudi Lozano-Rojas ${ }^{1,2, a}$, Emilio Cabello-Morales ${ }^{1,2, a, c}$, Herminio Hernádez-Diaz,2,b, \\ Cesar Loza-Munarriz',2,d
}

\begin{abstract}
RESUMEN
Objetivos. Determinar la prevalencia de sobrepeso y obesidad en adolescentes de un distrito urbano de Lima, Perú 2012. Materiales y métodos. Estudio transversal analítico, se incluyeron 1743 escolares de 12 a 17 años de edad seleccionados de diez instituciones educativas públicas y privadas, mediante una muestra por conglomerados, estratificado y aleatorio. En los escolares de las instituciones seleccionadas, se midió peso y talla. Para el diagnóstico de sobrepeso y obesidad se utilizó los criterios de la Organización Mundial de la Salud (OMS) y el Centers for Disease Control and Prevention (CDC). En el análisis de datos se empleó estadística descriptiva e inferencial. Resultados. Según los criterios de la OMS la prevalencia de sobrepeso fue 33,7\% (IC 95\%: 31,5-36,0) y obesidad 14,4\% (IC 95\%:12,8-16,1). Según criterios del CDC la prevalencia de sobrepeso fue $26,5 \%$ (IC 95\%: 24,4-28,6) y obesidad 13,9\% (IC 95\%: 12,3-15,6). La prevalencia de sobrepeso y obesidad fue significativamente mayor en varones de 12 y 13 años de edad e instituciones educativas privadas $(p<0,05)$. La concordancia entre los criterios de la OMS y el CDC para el diagnóstico de sobrepeso y obesidad fue muy buena. Conclusiones. Se encontró una alta prevalencia de sobrepeso y obesidad, con predominio en varones, escolares de 12 y 13 años e instituciones educativas privadas. Es necesario iniciar medidas de intervención que contribuyan a prevenir las enfermedades crónicas en la adultez, secundarias a la obesidad en la adolescencia.
\end{abstract}

Palabras clave: Obesidad; Sobrepeso; Prevalencia; Adolescente (fuente: DeCS BIREME).

\section{PREVALENCE OF OVERWEIGHT AND OBESITY IN ADOLESCENTS FROM AN URBAN DISTRICT OF LIMA, PERU 2012}

\begin{abstract}
Objectives. To determine the prevalence of overweight and obesity according to the criteria of the WHO and CDC in adolescents from an urban district of Lima, Peru 2012. Materials and methods. This cross-sectional study included 1,743 school children of 12 to 17 years of age selected from ten public and private educational institutions, using a randomized and stratified sample of "conglomerados" (neighborhoods). In the selected schools, weight and height were measured. For the diagnosis of overweight and obesity, criteria from the WHO and CDC were used. For data analysis, descriptive and inferential statistics were performed. Results. According to the WHO criteria, the prevalence of overweight was $33.7 \%(95 \% \mathrm{Cl} 31.5-36.0)$ and obesity was $14.4 \%$ (95\% Cl 12.8-16.1). According to CDC criteria, the prevalence of overweight was $26.5 \%$ (95\% Cl 24.4-28.6) and obesity was $13.9 \%$ (95\% Cl 12.3-15.6). The prevalence of overweight and obesity was significantly higher in males aged 12 and 13 years old and private educational institutions $(p<0.05)$. We found very good agreement between the WHO and CDC criteria for the diagnosis of overweight and obesity. Conclusions. A high prevalence of overweight and obesity was found, predominantly in male students between 12 and 13 years and private educational institutions. It is necessary to initiate intervention measures that can contribute to the prevention of chronic diseases in adulthood, secondary to obesity in adolescence.
\end{abstract}

Key words: Obesity; Overweight; Prevalence; Adolescent (source: MeSH NLM).

\section{INTRODUCCIÓN}

La obesidad es una enfermedad crónica no transmisible y un problema de salud pública mundial, que afecta a niños, adolescentes y adultos ${ }^{(1-5)}$. Se han identificado tres periodos críticos para su desarrollo: el prenatal, el periodo de 4 a 8 años de edad y la adolescencia ${ }^{(6)}$; los cuales constituyen factores de riesgo para desarrollar en la adultez: obesidad, enfermedades cardiovasculares, cerebrovasculares, diabetes mellitus tipo 2 y dislipidemia; aumentando su morbimortalidad ${ }^{(7,8)}$.

En EE.UU. la prevalencia de obesidad en adolescentes de 12 a 19 años, mostró un incremento del 5\% en 1980

\footnotetext{
Hospital Nacional Cayetano Heredia. Lima, Perú.

Facultad de Medicina, Universidad Peruana Cayetano Heredia. Lima, Perú

Medico endocrinólogo pediatra; ${ }^{\mathrm{b}}$ médico pediatra; ${ }^{\mathrm{c}}$ magíster en Salud Pública; ${ }^{\mathrm{d}}$ médico nefrólogo.

Recibido: 10-12-13 Aprobado: 09-07-14
}

Citar como: Lozano-Rojas G, Cabello-Morales E, Hernádez-Diaz H, Loza-Munarriz C. Prevalencia de sobrepeso y obesidad en adolescentes de un distrito urbano de Lima, Perú 2012. Rev Peru Med Exp Salud Publica. 2014;31(3):494-500. 
al $17,1 \%$ en el 2004 . El incremento de sobrepeso en la misma población fue hasta el 33,6\%, según el National Nutrition Health Examination Survey (NANHES) ${ }^{(9)}$. En los países latinoamericanos; diferentes estudios reportan incrementos de obesidad y sobrepeso en niños y adolescentes utilizando diferentes criterios. En Brasil, la prevalencia de obesidad y sobrepeso entre los 6 a 18 años aumentó del 4 al 13\% desde 1970 a $1997^{(10)}$. En Chile, la prevalencia de obesidad en escolares mostró un incremento del 6,5 al $13,1 \%$ en varones y del 7,7 al $14,7 \%$ en mujeres desde 1987 a 1996, y un incremento del 5 al $25 \%$ de la prevalencia global de obesidad en escolares de 6 a 16 años desde 1986 a $1996^{(11,12)}$. En Ecuador, la prevalencia global de obesidad y sobrepeso en escolares de 12-19 años fue $21,2 \%$ en el 2008 , siendo obesidad $7,5 \%$ y sobrepeso $13,7 \%$, y en mayor frecuencia en instituciones educativas privadas ${ }^{(13,14)}$.

En Perú, la información de la prevalencia de obesidad y sobrepeso en el período de la adolescencia es escasa. Según el informe de la Encuesta Nacional de Hogares (ENAHO) del 2008 la prevalencia de sobrepeso en mujeres de 10 a 19 años fue $15 \%$ y en varones $13,5 \%$, utilizando criterios de la Organización Mundial de la Salud (OMS) y no se diferenció obesidad de sobrepeso (15). Según la Encuesta Global de Salud Escolar del 2010, basados en el auto reporte anónimo de peso y talla, en escolares del segundo al cuarto año de secundaria en instituciones educativas públicas, la prevalencia de obesidad fue $3 \%$ y de sobrepeso $19,8 \%$, (16). El estudio de Pajuelo et al (17), en escolares mujeres de 9 a 17 años de una institución educativa pública de Lima, reportó la prevalencia de obesidad en $18 \%$ y sobrepeso en $17,2 \%$, utilizando los criterios de la OMS.

Por lo antes mencionado, en este estudio se planteó como objetivo determinar la prevalencia de sobrepeso y obesidad en escolares de 12 a17 años del distrito de Los Olivos en Lima, Perú según los criterios de la OMS y del Centers for Disease Control and Prevention (CDC).

\section{MATERIALES Y MÉTODOS}

\section{DISEÑO Y POBLACION DE ESTUDIO}

Se realizó un estudio transversal analítico, en una muestra de 1743 escolares de 12 a 17 años de edad, de ambos sexos, obtenida de diez Instituciones educativas de nivel secundario del distrito de Los Olivos. Se excluyeron, escolares con diagnóstico de hipotiroidismo, diabetes mellitus, síndrome de Cushing y cardiopatía, conocidos previamente por la institución educativa.

\section{SELECCIÓN DE LA MUESTRA}

La muestra fue por conglomerados estratificada, bietápica; en la primera etapa se seleccionó por muestreo estratificado con afijación proporcional, diez Instituciones educativas, tres públicas y siete privadas (7,5\% de 136 Instituciones educativas, 28 públicas y 108 privadas), las cuales fueron seleccionadas aleatoriamente; luego, en cada una de ellas se evaluó a todos los escolares que asistieron y aceptaron participar, no hubo negaciones para la participación. El tamaño de muestra calculado para este efecto fue 1180 escolares considerando una población de 32252 escolares del nivel secundario del distrito de Los Olivos, una prevalencia esperada de sobrepeso de $15 \%$ según ENAHO (15), un nivel de confianza del 95\%, precisión de $3 \%$, efecto de diseño de 2,0 y el $10 \%$ de tasas de no respuesta; al ejecutar el estudio se evaluó a 1743 escolares.

\section{PROCEDIMIENTOS}

A todos los escolares de las instituciones educativas seleccionadas se les determinó la edad decimal en base a la fecha de nacimiento y de evaluación, se midió el peso y talla, según los protocolos estándar de la OMS. El peso se midió con una balanza marca Seca, calibrada cada 5 tomas con $100 \mathrm{~g}$ de precisión. La talla se midió en posición de pie, descalzo; de las tres medidas se obtuvo un promedio. Las mediciones fueron realizadas por los investigadores y personal capacitado en diferentes horarios, por disponibilidad administrativa de las instituciones educativas. Se determinó el índice de masa corporal (IMC), obtenido del cociente peso en kilogramos entre talla elevada al cuadrado.

El diagnóstico de sobrepeso y obesidad se realizó según los criterios de la OMS 2006, que define sobrepeso si el IMC es mayor de +1 y menor o igual de +2 desviaciones estándar (DE) y obesidad si el IMC es mayor de $+2 \mathrm{DE}$, para la edad y sexo correspondiente (1) y del CDC 2000 que define sobrepeso si el IMC está entre el 85 y menor del 95 percentil y obesidad si el IMC es mayor o igual al 95 percentil, para la edad y sexo correspondiente ${ }^{(2)}$.

Se consideró variable dependiente la presencia o ausencia de sobrepeso u obesidad, y como variables independientes edad, sexo y tipo de institución educativa a la que pertenecen los escolares. La tasa de prevalencia se calculó considerando como numerador los casos de sobrepeso u obesidad y como denominador el total de escolares participantes en el estudio. 
Tabla 1. Características antropométricas según sexo en escolares de 12 a 17 años de un distrito urbano de Lima, Perú 2012

\begin{tabular}{|c|c|c|c|c|c|c|c|}
\hline & \multicolumn{3}{|c|}{ Varones } & \multicolumn{3}{|c|}{ Mujeres } & \multirow{2}{*}{$p^{*}$} \\
\hline & Media & DE & (IC 95\%) & Media & $\mathrm{DE}$ & (IC 95\%) & \\
\hline \multicolumn{8}{|l|}{12 años } \\
\hline Peso & 55,1 & 12,8 & $(53,0-57,2)$ & 52,5 & 9,7 & $(51,1-53,9)$ & 0,035 \\
\hline Talla & 154,4 & 8,1 & $(153,0-155,7)$ & 152,2 & 5,5 & $(151,4-152,9)$ & 0,004 \\
\hline IMC & 23,0 & 4,4 & $(22,3-23,7)$ & 22,6 & 3,6 & $(22,1-23,1)$ & 0,401 \\
\hline \multicolumn{8}{|l|}{13 años } \\
\hline Peso & 55,9 & 11,8 & $(54,2-57,6)$ & 52,6 & 9,3 & $(51,1-53,9)$ & 0,004 \\
\hline Talla & 157,9 & 7,5 & $(156,8-159,0)$ & 153,4 & 5,3 & $(152,6-154,3)$ & 0,001 \\
\hline IMC & 22,3 & 4,0 & $(21,7-22,9)$ & 22,3 & 3,6 & $(21,7-22,8)$ & 0,957 \\
\hline \multicolumn{8}{|l|}{14 años } \\
\hline Peso & 59,7 & 10,9 & $(57,9-61,4)$ & 56,4 & 10,4 & $(54,8-58,1)$ & 0,008 \\
\hline Talla & 163,1 & 5,9 & $(162,2-164,1)$ & 155,2 & 5,2 & $(154,4-156,1)$ & 0,001 \\
\hline IMC & 22,3 & 3,5 & $(21,8-22,9)$ & 23,4 & 4,0 & $(22,7-24,0)$ & 0,025 \\
\hline \multicolumn{8}{|l|}{15 años } \\
\hline Peso & 64,8 & 14,6 & $(62,7-66,9)$ & 57,2 & 9,3 & $(55,8-58,5)$ & 0,001 \\
\hline Talla & 166,1 & 5,7 & $(165,3-166,9)$ & 155,5 & 5,1 & $(154,7-156,2)$ & 0,001 \\
\hline IMC & 23,4 & 4,6 & $(22,7-24,1)$ & 23,6 & 3,4 & $(23,1-24,1)$ & 0,591 \\
\hline \multicolumn{8}{|l|}{16 años } \\
\hline Peso & 63,8 & 11,9 & $(61,9-65,8)$ & 57,3 & 8,9 & $(55,9-58,8)$ & 0,001 \\
\hline Talla & 165,7 & 5,6 & $(164,8-166,6)$ & 155,4 & 6,0 & $(154,4-156,4)$ & 0,001 \\
\hline IMC & 23,2 & 3,8 & $(22,6-23,8)$ & 23,7 & 3,3 & $(23,2-24,3)$ & 0,216 \\
\hline \multicolumn{8}{|l|}{17 años } \\
\hline Peso & 63,3 & 11,5 & $(59,9-66,6)$ & 56,1 & 8,1 & $(53,6-58,5)$ & 0,001 \\
\hline Talla & 166,8 & 6,1 & $(165,0-168,5)$ & 154,8 & 6,1 & $(153,0-156,7)$ & 0,001 \\
\hline IMC & 22,7 & 3,6 & $(21,7-23,7)$ & 23,4 & 2,7 & $(22,5-24,2)$ & 0,331 \\
\hline
\end{tabular}

DE: desviación estándar, IMC: índice de masa corporal, *prueba t de Student

\section{ANÁLISIS ESTADÍSTICO}

Se empleó estadística descriptiva para las características demográficas y antropométricas, y la prueba de ShapiroWilks para evaluar el tipo de distribución de las variables continuas. Para la comparación de prevalencias de sobrepeso y obesidad según los criterios de OMS y CDC por edad, sexo e institución educativa se empleó la prueba $Z$ de diferencia de proporciones Para determinar la concordancia entre los criterios de la OMS y CDC se empleó el índice de concordancia Kappa. Para relacionar variables categóricas se empleó la prueba chi cuadrado. Para relacionar variables continuas con distribución normal y homogeneidad de varianzas se empleó t de Student para muestras independientes. En el análisis multivariado entre la presencia de sobrepeso u obesidad y las variables independientes se empleó el modelo de regresión logística múltiple, para investigar el efecto de pertenecer a una institución pública o privada, el incremento de 1 unidad de edad y el sexo en la variable dependiente. Se calculó el intervalo de confianza del $95 \%$ se consideró el valor de $p<0,05$ como nivel de significancia estadística. El análisis de datos se realizó con el programa estadístico Stata 11 (Stata Corp., TX, USA).

\section{CONSIDERACIONES ÉTICAS}

El estudio fue aprobado por el Comité de Ética de la Universidad Peruana Cayetano Heredia. Se obtuvo el consentimiento de los padres o tutores a través de la dirección de cada institución educativa y el asentimiento de cada escolar.

\section{RESULTADOS}

Se incluyeron en el estudio 1743 escolares, de los cuales el 50,1\% fueron mujeres. La media de edad fue $14,6 \pm 1,5$ años. El $65,2 \%$ de los escolares pertenecían a instituciones educativas privadas y el $34,8 \%$ a públicas. En cada grupo de edad, la media del peso y talla según sexo fue estadísticamente diferente $(p<0,05)$. La media del IMC fue similar en ambos sexos, excepto a los 14 años de edad (Tabla 1).

La prevalencia de sobrepeso según el criterio de la OMS fue $33,7 \%$ (IC 95\%: 31,5-36,0) y obesidad $14,4 \%$ (IC $95 \%: 12,8-16,1)$. Según el criterio del CDC la prevalencia de sobrepeso fue $26,5 \%$ (IC 95\%: $24,4-28,6$ ) y obesidad 
Tabla 2. Prevalencia de sobrepeso y obesidad según criterios de la OMS y CDC en escolares de 12 a 17 años de un distrito urbano de Lima, Perú 2012

\begin{tabular}{lccccccc}
\hline & \multicolumn{2}{c}{ OMS } & & \multicolumn{2}{c}{ CDC } \\
\cline { 2 - 3 } \cline { 5 - 6 } & $\%$ & (IC 95\%) & & $\%$ & (IC 95\%) & $\boldsymbol{p}^{*}$ \\
\hline Bajo peso & 2,3 & $(1,6-3,1)$ & & 1,5 & $(0,9-2,2)$ & 0,083 \\
Normal & 49,6 & $(47,2-51,9)$ & & 58,2 & $(55,8-60,5)$ & 0,001 \\
Sobrepeso & 33,7 & $(31,5-36,0)$ & & 26,5 & $(24,4-28,6)$ & 0,001 \\
Obesidad & 14,4 & $(12,8-16,1)$ & & 13,9 & $(12,3-15,6)$ & 0,666 \\
\hline
\end{tabular}

* Prueba $Z$ para diferencia de proporciones

13,9\% (IC 95\%: 12,3-15,6). La comparación de prevalencias, mediante la prueba $Z$ para diferencia de proporciones, mostró diferencia significativa $(p=0,001)$ entre los criterios de la OMS y CDC para sobrepeso y no para obesidad (Tabla 2).

La concordancia entre los criterios de la OMS y CDC para el diagnóstico de bajo peso, peso normal, sobrepeso y obesidad fue muy buena (Kappa global $=84,7 \% ; p=0,001$ ) asi como la concordancia individual para el diagnóstico de obesidad, sobrepeso y peso normal ( $k=97,4 \%$; $82,8 \%$ y $80,1 \%$ respectivamente). Sin embargo para evaluar bajo peso fue buena $(\mathrm{k}=78,4 \%)$. La confiabilidad interevaluadores fue $88,1 \%$.

El análisis de la prevalencia de sobrepeso y obesidad según los criterios de la OMS y CDC mostró una tendencia decreciente con el incremento de la edad en ambos sexos. La comparación entre ambos criterios, en los varones la prevalencia de sobrepeso en cada grupo de edad es estadísticamente similar, excepto a los 14 años de edad. La prevalencia de obesidad en cada grupo de edad es estadísticamente similar con los criterios de la OMS y CDC. En las mujeres la prevalencia de sobrepeso y obesidad según criterios de OMS y CDC en cada grupo de edad son estadísticamente similares (Tabla 3).

La prevalencia de obesidad según sexo utilizando los criterios de la OMS fue 16,9\% (IC 95\%: 14,4-19,5) en los varones y $11,9 \%$ (IC 95\%: 9,8-14,3) en las mujeres, y de sobrepeso 32,6\% (IC 95\%: 29,5-35,8) en los varones y $34,9 \%$ (IC 95\%: 31,7-38,1) en las mujeres. Utilizando los criterios del CDC la prevalencia de obesidad fue 15,8\% (IC 95\%: 13,4-18,4) en los varones y 11,9\% (IC 95\%: $9,8-14,3$ ) en las mujeres y de sobrepeso fue $24,5 \%$ (IC $95 \%$ : $21,6-27,5$ ) en los varones y $28,4 \%$ (IC 95\%: $25,5-$ $31,6)$ en las mujeres. La comparación de prevalencias de obesidad y sobrepeso según sexo mostró que los varones tienen mayor probabilidad de tener obesidad que las mujeres, con una diferencia significativa y no se encontró diferencias por sexo entre las prevalencias de sobrepeso según ambos criterios (Tabla 4).

Según tipo de institución educativa, la prevalencia de sobrepeso utilizando los criterios de la OMS fue $37,1 \%$ (IC 95\%: 34,3-40,0) en las I.E. privadas y 27,4\% (IC 95\%: 23,9-31,1) en las instituciones educativas públicas y de obesidad fue 16,8\% (IC 95\%: 14,7-19,1) en las privadas y del 9,9\% (IC 95\%: 7,6-12,6) en las I.E. públicas. Utilizando los criterios del CDC la prevalencia de sobrepeso fue 29,3\% (IC 95\%: 26,7-32,0) en las I.E. privadas y del $21,1 \%$ (IC 95\%: 17,9-24,6) en las públicas y de obesidad fue $16,2 \%$ (IC 95\%; 14,1-18,5) en las I.E. privadas y $9,6 \%$ (IC 95\%: 7,3-12,2) en las I.E. públicas

Tabla 3. Prevalencia de sobrepeso y obesidad por edad y sexo en escolares de 12 a 17 años de un distrito urbano de Lima, Perú 2012

\begin{tabular}{|c|c|c|c|c|c|c|c|c|c|c|c|}
\hline \multirow{3}{*}{$\begin{array}{l}\text { Edad } \\
\text { según } \\
\text { sexo }\end{array}$} & \multicolumn{5}{|c|}{ OMS } & \multicolumn{4}{|c|}{$\mathrm{CDC}$} & \multirow{3}{*}{$p^{*}$} & \multirow{3}{*}{$p \mp$} \\
\hline & & Bajo peso & Normal & Sobrepeso & Obesidad & Bajo peso & Normal & Sobrepeso & Obesidad & & \\
\hline & $\mathbf{N}$ & $\%$ (IC 95\%) & $\%$ (IC 95\%) & $\%$ (IC 95\%) & $\%$ (IC 95\%) & $\%$ (IC 95\%) & $\%$ (IC 95\%) & $\%$ (IC 95\%) & $\%$ (IC 95\%) & & \\
\hline \multicolumn{12}{|c|}{ Varones } \\
\hline 12 & 147 & $0,7(0,0-3,7)$ & $27,2(20,2-35,2)$ & $38,1(30,2-46,5)$ & $34,0(26,4-42,3)$ & 0 & $38,1(30,2-46,4)$ & $32,7(25,2-40,9)$ & $29,3(22,0-37,3)$ & 0,333 & 0,386 \\
\hline 13 & 185 & $1,6(0,3-4,7)$ & $42,7(35,5-50,2)$ & $37,3(30,3-44,7)$ & $18,4(13,1-24,7)$ & $1,1(0,1-3,8)$ & $53,0(45,5-60,3)$ & $28,7(22,2-35,7)$ & $17,3(12,1-23,5)$ & 0,078 & 0,782 \\
\hline 14 & 158 & $1,3(0,2-4,5)$ & $51,3(43,2-59,3)$ & $34,8(27,4-48,2)$ & $12,7(7,9-18,9)$ & $1,3(0,2-4,5)$ & $62,7(54,6-70,2)$ & $23,4(17,1-30,8)$ & $12,7(7,9-18,9)$ & 0,025 & 1,000 \\
\hline 15 & 182 & $5,5(2,7-9,9)$ & $50,6(43,1-58,0)$ & $29,7(3,1-36,9)$ & $14,3(9,5-20,2)$ & $4,4(1,9-8,4)$ & $60,4(52,9-67,6)$ & $20,9(15,2-27,5)$ & $14,3(9,5-20,2)$ & 0,053 & 1,000 \\
\hline 16 & 150 & $4,0(1,5-8,5)$ & $58,7(50,3-66,3)$ & $29,3(22,2-37,3)$ & $8,0(4,2-13,6)$ & $2,0(0,4-5,7)$ & $68,0(59,9-75,4)$ & $22,0(15,7-29,5)$ & $8,0(4,2-13,6)$ & 0,147 & 1,000 \\
\hline 17 & 49 & $6,1(1,3-16,8)$ & $71,4(56,7-83,4)$ & $12,2(4,6-24,7)$ & $10,2(3,4-22,2)$ & $4,1(0,4-13,9)$ & $77,6(63,4-88,2)$ & $8,2(2,3-19,6)$ & $10,2(3,4-22,2)$ & 0,513 & 1,000 \\
\hline \multicolumn{12}{|c|}{ Mujeres } \\
\hline 12 & 183 & $0,6(0,0-3,0)$ & $37,7(30,7-45,1)$ & $42,1(34,8-49,6)$ & $19,7(14,2-26,2)$ & $0,6(0,0-3,0)$ & $45,9(38,5-53,4)$ & $34,3(27,6-41,8)$ & $19,1(13,7-25,6)$ & 0,129 & 0,884 \\
\hline 13 & 164 & $3,1(0,9-6,9)$ & $45,7(37,9-53,7)$ & $39,6(32,1-47,6)$ & $11,6(7,1-17,5)$ & $2,4(0,6-6,1)$ & $54,9(46,9-62,6)$ & $30,5(23,5-38,1)$ & $12,2(7,6-18,2)$ & 0,084 & 0,866 \\
\hline 14 & 150 & $0,7(0,0-3,7)$ & $56,0(47,7-64,1)$ & $29,3(22,2-37,3)$ & $14,0(8,9-20,6)$ & 0 & $61,3(53,0-69,1)$ & $24,7(18,0-32,4)$ & $14,0(8,9-20,6)$ & 0,369 & 1,000 \\
\hline 15 & 183 & $2,2(0,5-5,5)$ & $54,1(46,6-61,5)$ & $34,4(27,6-41,8)$ & $9,3(5,5-14,5)$ & 0 & $61,8(54,3-68,8)$ & $29,0(22,5-36,1)$ & $9,3(5,5-14,5)$ & 0,267 & 1,000 \\
\hline 16 & 147 & $1,4(0,2-4,8)$ & $62,6(54,2-70,4)$ & $28,6(21,4-36,6)$ & $7,5(3,8-12,9)$ & $1,4(0,2-4,8$ & $68,0(59,8-75,5)$ & $23,1(16,6-30,8)$ & $7,5(3,8-12,9)$ & 0,281 & 1,000 \\
\hline 17 & 45 & $4,1(0,5-15,1)$ & $66,7(51,0-79,9)$ & $28,9(16,4-44,3)$ & 0 & $0,4(0,5-15,1)$ & $71,1(55,7-83,6)$ & $24,4(12,9-39,5)$ & 0 & 0,629 & 1,000 \\
\hline
\end{tabular}

* Valor de p para la diferencia de porcentajes de sobrepeso, prueba Z para diferencia de proporciones

$\mp$ Valor de $p$ para la diferencia de porcentajes de obesidad, prueba $Z$ para diferencia de proporciones 
Tabla 4. Prevalencia de sobrepeso y obesidad según sexo en escolares de 12 a 17 años de un distrito urbano de Lima, Perú 2012

\begin{tabular}{|c|c|c|c|c|c|c|c|c|c|c|}
\hline & \multicolumn{4}{|c|}{ Varones $(n=871)$} & \multicolumn{4}{|c|}{ Mujeres $(n=872)$} & \multirow{3}{*}{$p^{*}$} & \multirow{3}{*}{$p \mp$} \\
\hline & Bajo peso & Peso normal & Sobrepeso & Obesidad & Bajo peso & Peso normal & Sobrepeso & Obesidad & & \\
\hline & $\%$ (IC 95\%) & \% (IC 95\%) & $\%$ (IC 95\%) & $\%$ (IC 95\%) & $\%$ (IC 95\%) & $\%$ (IC 95\%) & $\%$ (IC 95\%) & $\%$ (IC 95\%) & & \\
\hline OMS & $2,9(1,8-4,2)$ & $47,7(44,3-51,0)$ & $32,6(29,5-35,8)$ & $16,9(14,4-19,5)$ & $1,7(0,9-2,8)$ & $51,5(48,1-54,9)$ & $34,9(31,7-38,1)$ & $11,9(9,8-14,3)$ & 0,309 & 0,002 \\
\hline CDC & $2,0(1,1-3,1)$ & $57,8(54,3-61,1)$ & $24,5(21,6-27,5)$ & $15,8(13,4-18,4)$ & $1,0(0,4-1,9)$ & $58,6(55,2-61,9)$ & $28,4(25,5-31,6)$ & $11,9(9,8-14,3)$ & 0,065 & 0,018 \\
\hline
\end{tabular}

* Valor de $p$ para la diferencia de porcentajes de sobrepeso, prueba Z para diferencia de proporciones.

$\mp$ Valor de $p$ para la diferencia de porcentajes de obesidad prueba Z para diferencia de proporciones.

Tabla 5. Prevalencia de sobrepeso y obesidad según tipo de instituciones educativas en escolares de 12 a 17 años de un distrito urbano de Lima, Perú 2012

\begin{tabular}{|c|c|c|c|c|c|c|c|c|c|c|}
\hline & \multicolumn{4}{|c|}{ Institución educativa pública ( $n=606$ ) } & \multicolumn{4}{|c|}{ Institución educativa privada $(n=1137)$} & \multirow{3}{*}{$p^{*}$} & \multirow{3}{*}{$p \mp$} \\
\hline & Bajo peso & Peso normal & Sobrepeso & Obesidad & Bajo peso & Peso normal & Sobrepeso & Obesidad & & \\
\hline & $\%($ IC 95\%) & $\%$ (IC 95\%) & $\%$ (IC 95\%) & $\%($ IC 95\%) & $\%$ (IC 95\%) & $\%$ (IC 95\%) & $\%$ (IC 95\%) & $\%$ (IC 95\%) & & \\
\hline OMS & $2,5(1,4-4,0)$ & $60,2(56,2-64,2)$ & $27,4(23,9-31,1)$ & $9,9(7,6-12,6)$ & $2,2(1,4-3,2)$ & $43,9(40,9-46,8)$ & $37,1(34,3-40,0)$ & $16,8(14,7-19,1)$ & 0,001 & 0,001 \\
\hline $\mathrm{CDC}$ & $1,6(0,8-3,0)$ & $67,7(63,8-71,4)$ & $21,1(17,9-24,6)$ & $9,6(7,3-12,2)$ & $1,4(0,8-2,3)$ & $53,1(50,2-56,1)$ & $29,3(26,7-32,0)$ & $16,2(14,1-18,5)$ & 0,001 & 0,001 \\
\hline
\end{tabular}

* Valor de $p$ para la diferencia de porcentajes de sobrepeso, prueba Z para diferencia de proporciones.

$\mp$ Valor de $p$ para la diferencia de porcentajes de obesidad prueba $Z$ para diferencia de proporciones.

(Tabla 5). La comparación de prevalencias de sobrepeso y obesidad entre I.E. privadas y públicas mostró diferencias significas, con ambos criterios, de la OMS y CDC; siendo mayor en las I.E. privadas (Tabla 5).

En el análisis multivariado, el modelo de regresión logística mostró que los escolares que pertenecen a una I.E. privada tuvieron mayor riesgo de sobrepeso OR 1,70 (IC 95\%: 1,36-2,14) y obesidad OR 1,65 (IC $95 \%: 1,21-2,27)$, el aumento de una unidad de la edad en años es un factor protector para el desarrollo de sobrepeso OR 0,79 (IC 95\%: 0,73-0,85) u obesidad OR 0,72 (IC 95\%: 0,65-0,79), ser mujer es un factor protector para el desarrollo de obesidad OR 0,63 (IC $95 \%$ : $0,48-0,83)$ pero no es significativo para sobrepeso (Tabla 6).

Tabla 6. Asociación entre sobrepeso y obesidad con el tipo de instituciones, edad y sexo en escolares de 12 a 17 años de un distrito urbano de Lima, Perú 2012

\begin{tabular}{lccc}
\hline & OR & (IC 95\%) & p \\
\hline Sobrepeso & & & \\
I.E. privadas & 1,70 & $(1,36-2,14)$ & 0,001 \\
Edad & 0,79 & $(0,73-0,85)$ & 0,001 \\
Sexo femenino & 0,98 & $(0,79-1,20)$ & 0,882 \\
Obesidad & & & \\
I.E privadas & 1,65 & $(1,21-2,27)$ & 0,002 \\
Edad & 0,72 & $(0,65-0,79)$ & 0,001 \\
Sexo femenino & 0,63 & $(0,48-0,83)$ & 0,001 \\
\hline
\end{tabular}

I.E: institución educativa. Progresión logística múltiple.

\section{DISCUSIÓN}

Este estudio muestra una alta prevalencia de sobrepeso y obesidad en escolares de 12 a 17 años de edad en un distrito urbano de Lima, tanto con los criterios de la OMS y el CDC, comparado con lo reportado por la ENAHO $2008{ }^{(15)}$, la Encuesta Global de Salud Escolar (16) y el estudio de Pajuelo et al. en el 2009 (17). Aunque es difícil hacer comparaciones con la prevalencia reportada en otros países debido a la diversidad de metodologías y criterios utilizados para el diagnóstico, los resultados encontrados son casi similares a la prevalencia de obesidad y sobrepeso en adolescentes de 12 a 19 años de EE. UU. en el 2003-2004 (11,14). Por lo cual, aproximadamente uno de cada dos o tres escolares de la población estudiada presentan obesidad o sobrepeso. Considerando que el distrito de Los Olivos es el octavo más poblado de Lima y en intenso crecimiento urbano y comercial (20), es necesario tomar medidas de intervención inmediatas para prevenir las enfermedades crónicas prevenibles en la adultez, secundarias a la obesidad en el niño y el adolescente.

La comparación de la prevalencia de sobrepeso y obesidad estimada según los criterios de la OMS 2006 y CDC 2000, mostraron mayor prevalencia de sobrepeso cuando se utiliza los criterios de la OMS, pero sin diferencia estadística para la determinación de la prevalencia de obesidad. Estos resultados son similares a los encontrados por Pajuelo et al. cuando refiere que la prevalencia global de sobrepeso y obesidad es mayor al utilizar los criterios de la OMS ${ }^{(17,18)}$. Según el presente estudio solo se encontró diferencias entre ambos criterios 
para la prevalencia de sobrepeso pero no para obesidad, probablemente debido que los puntos de corte para la identificación de sobrepeso son más bajos según la OMS. Sin embargo, el análisis de concordancia global entre ambos criterios es muy buena, siendo mejor para evaluar obesidad; esto debería tenerse en consideración al realizar comparaciones entre diferentes estudios y durante la evaluación de los pacientes, debido a que cierta población con sobrepeso puede no ser identificada según los criterios que se utilice, por lo tanto podría sugerirse que en aquellos escolares que tienen factores de riesgo adicional para otras enfermedades crónicas no transmisibles, se utilice los criterios de la OMS, que permitan la detección de sobrepeso y una intervención precoz.

La prevalencia de sobrepeso y obesidad fue mayor a los 12 años de edad en ambos sexos según los criterios de OMS y CDC y mostrando una tendencia decreciente a mayor edad, esto reafirma el concepto que la adolescencia es una etapa crítica para el desarrollo de obesidad ${ }^{(6)}$, poniendo en evidencia que podría ser los primeros años de la adolescencia los de mayor riesgo, lo cual es importante considerarlo para las medidas de intervención en este rango etario.

La prevalencia de obesidad fue mayor en los varones, mientras que el sobrepeso fue similar entre ambos sexos, estos resultados concuerdan con el estudio de Yépez et al. ${ }^{(14)}$, lo que confirma que el patrón de adiposidad no solo es dependiente del sexo, sino también influenciado por otros factores como el grado de desarrollo puberal y la raza ${ }^{(21)}$.

La mayor prevalencia de sobrepeso y obesidad encontrada en las I.E. privadas utilizando ambos criterios, mostró diferencia significativa, similar a los datos reportados en Ecuador ${ }^{(14)}$. Por lo cual, se puede plantear probables factores de riesgo como: diferente sistema educativo, que conlleva a diferentes estilos de actividad física o sedentarismo en los escolares, mayor consumo de "comida chatarra" que son alimentos con pobre aporte de nutrientes, alto aporte calórico por una elevada cantidad de azúcar, sal y/o grasas trans y/o grasas saturadas, aditivos químicos y saborizantes artificiales, así como al diferente poder adquisitivo que condicione otorgar propina al escolar, que conlleva a la compra de alimentos de alto contenido energético y pobre contenido nutricional.

Los resultados reportados en este estudio ponen en evidencia la magnitud del sobrepeso y la obesidad en adolescentes como un problema de salud pública, por lo cual es importante y urgente que se efectivicen medidas de intervención y prevención que sensibilicen a la población respecto a la obesidad y sobrepeso, se difundan y cumplan medidas correctivas. Entre las planteadas por el estado se tiene la Ley de Promoción de la Alimentación Saludable para Niños, Niñas y Adolescentes ${ }^{(19)}$, la cual plantea la promoción de la educación nutricional, quioscos y comedores saludables, deporte y actividad física, así como la vigilancia publicitaría de alimentos y bebidas no alcohólicas. Sin bien el estudio presenta limitaciones propias de un estudio con diseño de tipo transversal, la principal fortaleza es el relativo gran conjunto de datos pero solo se limitan a datos de un distrito de Lima.

En conclusión, se encontró una alta prevalencia de sobrepeso y obesidad, con predominio en varones, escolares entre 12 a 13 años e instituciones educativas privadas. La concordancia entre los criterios de la OMS y el CDC para el diagnóstico de sobrepeso y obesidad fue muy buena. No se encontró diferencia significativa en la prevalencia de obesidad, pero sí de sobrepeso cuando se compararon los criterios de diagnóstico de la OMS y CDC.

Contribuciones de autoría. GL participó en la concepción, diseño del artículo, recolección de resultados, análisis e interpretación de datos, redacción y revisión crítica del artículo. EC participó en la concepción, diseño del artículo, análisis e interpretación de datos, revisión crítica del artículo. HH participó del diseño del estudio y revisión crítica del artículo. CL realizó la asesoría estadística y revisión crítica del artículo. Todos los autores aprobaron la versión final a publicar.

Fuentes de financiamiento: autofinanciado

Conflicto de Intereses: los autores declaran no tener conflictos de interés.

\section{REFERENCIAS BIBLIOGRÁFICAS}

1. WHO Europe, ENHIS. Prevalence of excess body weight and obesity in children and adolescents. Geneva: WHO; 2007.

2. Centers for Disease Control and Prevention. Overweight and obesity [internet]. Atlanta: CDC; 2007 [citado el 10 de diciembre del 2013]. Disponible en: http://www.cdc.gov/ obesity/

3. Delgado-Noguera M, Tort $S$, Bonfill X, Gich I, Alonso-Coello P. Quality assessment of clinical practice guidelines for the prevention and treatment of childhood overweight and obesity. Eur J Pediatr. 2009 Jul;168(7):789-99. doi: 10.1007/ s00431-008-0836-5.

4. World Health Organization. Cause specific mortality and morbidity. En: World Health Organization. World 
Health Statistics 2010. Ginebra: WHO; 2010. p. 59-72.

5. World Health Organization. Preventing chronic diseases: a vital investment. WHO global report [internet]. Ginebra: WHO; 2005. [citado el 10 de diciembre de 2013]. Disponible en: http://www.who.int/ chp/chronic_disease_report/en/

6. Dietz W. Critical periods in childhood for the development of obesity. Am J Clin Nutr. 1994 May;59(5):955-9.

7. Hayman LL, Williams CL, Daniels SR, Steinberger J, Paridon S, Dennison BA, et al. Cardiovascular health promotion in the schools: a statement for health and education professionals and child health advocates from the Committee on Atherosclerosis, Hypertension, and Obesity in Youth (AHOY) of the Council on Cardiovascular Disease in the Young, American Heart Association. Circulation. 2004 Oct 12;110(15):2266-75.

8. York DA, Rössner S, Caterson I, Chen CM, James WP, Kumanyika S. Prevention Conference VII: Obesity, a worldwide epidemic related to heart disease and stroke: Group I: worldwide demographics of obesity. Circulation. 2004 Nov 2;110(18):e463-70.

9. Ogden CL, Carroll MD, Curtin LR, McDowell MA, Tabak CJ, Flegal KM. Prevalence of overweight and obesity in the United States, 1999-2004. JAMA. 2006 Apr;295(13):1549-55.

10. OPS (Organización Panamericana de la Salud). Régimen alimentario, nutrición y actividad física. Washington DC: OPS. 2003.

11. Instituto de Nutrición y Tecnología de los Alimentos. Obesidad desde la infancia. Santiago de Chile: INTA; 2005.

12. Muzzo S, Cordero J, Burrows R. Cambios en la prevalencia en del exceso de peso del escolar chileno en los últimos 8 años. Rev Chi Nutr. 1999 Dic;26(3):311-5.

13. Yépez R. La obesidad en el Ecuador en tempranas edades de la vida. Revista de la Facultad de Ciencias Médicas. 2005;30:20-4.

14. Yépez R, Carrasco F, Baldeon $\mathrm{M}$. Prevalencia de sobrepeso y obesidad en estudiantes adolescentes ecuatorianos del área urbana. Arch Latinoam Nutr. 2008 Jun;58(2):139-43.

15. Instituto Nacional de Estadística e Informática (INEI). Encuesta Nacional de Hogares. 1er Trimestre 2008. Lima: INEI; 2008.

16. Perú. Ministerio de Salud. Encuesta global de salud escolar. Resultados Perú 2010. Lima: MINSA; 2011.

17. Pajuelo J, Medrano M. El uso de diferentes poblaciones referenciales en el diagnóstico de los principales problemas nutricionales en niños y adolescentes. An Fac Med. 2009;70(3):193-8.

18. Pajuelo J, Mosquera Z, Quiroz R, Santolalla M. El sobrepeso y la obesidad en adolescentes. Diagnóstico. 2003;42(1):17-22.

19. Ministerio de Educación. Ley $\mathrm{N}^{\mathrm{o}}$ 30021. Ley de Promoción de la alimentación saludable para niños, niñas y adolescentes. El Peruano 2013 May 17;494937-9.

20. Instituto Nacional de Estadística e Informática. Perfil sociodemográfico de la provincia de Lima. Censos Nacionales 2007: XI de Población y VI de Vivienda. Lima: INEI; 2008.

21. Daniels SR, Khoury PR, Morrison JA. Utility of different measures of body fat distribution in children and adolescents. Am J Epidemiol. 2000 Dec;152(12):1179-84.

Correspondencia: Gaudi Lozano Rojas Dirección: Unidad de Endocrinología Pediátrica, Hospital Nacional Cayetano Heredia, Av. Honorio Delgado, Lima 31, Perú.

Teléfono: 943774584

Correoelectrónico:gelr@hotmail.com

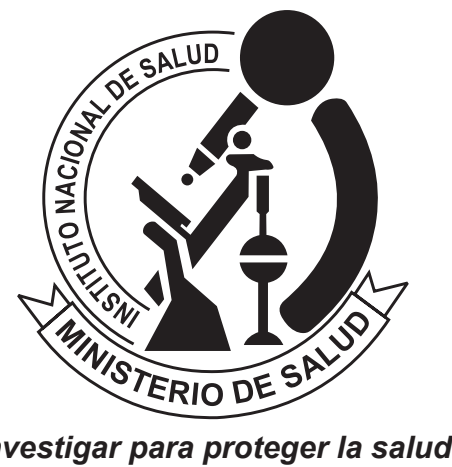

REVISTA PERUANA DE MEDICINA EXPERIMENTAL Y SALUD PÚBLICA CUMPLIENDO SUS METAS Y PROYECTÁNDOSE AL FUTURO

\section{Visite los contenidos de la revista en: www.ins.gob.pe/rpmesp}

